\title{
Correction to: Genetically Engineered Probiotics
}

Saba Haq and Naresh Poondla

\section{Correction to:}

Chapter 14 in: P. K. Deol (ed.), Probiotic Research in Therapeutics, https://doi.org/10.1007/978-981-15-8214-1_14

The first author's name of this chapter has been updated as "Saba Haq". 